\title{
Combining Facial Skin Mark and Eigenfaces for Face Recognition
}

\author{
Zhi Zhang, Sergey Tulyakov, and Venu Govindaraju \\ Center for Unified Biometrics and Sensors (CUBS) \\ University at Buffalo, USA
}

\begin{abstract}
In this paper we investigate the use of facial skin mark information for biometric person verification. We performed statistical analysis of the facial skin mark information. The position, size and color intensity of the skin marks are considered as features for skin mark based face matching. Developed facial skin mark matcher has good performance, but can not be applied to faces with no detected skin marks. Due to non-universality of skin mark information, a novel combination algorithm of traditional Eigenfaces matcher with skin mark matcher has been proposed. The resulting combined face matcher has universality property and delivers better performance than either single matcher. The AR Face Database was used in experiments.
\end{abstract}

\section{Introduction}

In order to process ultraviolet radiation human skin produces a pigment, melanin. A localized concentration of melanin in the skin can form into a mole or a freckle, termed as a melanocytic naevus. Nevi, as well as, other small scale structures on the skin surface such as wrinkles or scars, have different appearance from normal skin regions. With their relatively stable appearance, these irregularities, especially prominent moles, freckles or scars, in the face region present potential valuable information, and therefore can facilitate the task of face recognition. In particular, the skin irregularities have been used by law enforcement agencies for purpose of personal identification. In this paper we study the benefits of using skin mark information for the automatic face recognition.

Most of the current face recognition systems are designed to process faces holistically. One of the most prominent examples of holistic matching algorithms, the Eigenfaces approach [1], which introduces PCA on the raw image data to reduce feature dimension, therefore implicitly treats local variations as noise. Similar approaches include Fisherfaces [2]. Other model based systems, such as Active Appearance Model in $2 D$ [3], or the Morphable Model in $3 D$ [4] use PCA to model intra-class variations. Small unexpected details cannot be captured by these methods. Many existing algorithms utilize textons, DCT coefficients or Gabor wavelet features to represent local facial skin information, but there are no explicit representations of aforementioned facial skin marks.

Only limited research has been done so far on exploiting skin marks for face recognition. The work by Lin et al. [5] utilizes a multilayer representation of a face with global appearance, facial features, skin texture and irregularities. A SIFT framework

M. Tistarelli and M.S. Nixon (Eds.): ICB 2009, LNCS 5558, pp. 424 433, 2009.

(C) Springer-Verlag Berlin Heidelberg 2009 
is utilized to detect and describe details of irregular skin regions, which are combined into an elastic graph for recognition. Based on the $3 D$ Morphable Model developed in [4], the work of Pierrard et al. [6] presented a framework to localize prominent skin irregularities, mainly moles, in facial skin regions. Their system detects potential moles with a sensitive multi-scale template matching algorithm. Using skin segmentation and local saliency measurement, the candidates are filtered based on their discriminative potential.

The goal of this paper is to present study of facial skin marks as a possible biometric trait for the task of personal identification. Statistical analysis has been done on the characteristics of facial skin marks, such as universality and stability. We developed a facial skin mark matching algorithm, which has good performance. However, due to the nature of facial skin mark, it does not have perfect universality. To address this issue, one novel approach combining conventional Eigenfaces algorithm with our facial skin mark matcher was presented, which has universality property and delivers better performance than either one of the algorithm alone.

The organization of this paper is as follows: In Section 2, the statistical analysis on the characteristics of facial skin marks and their detection, localization, and feature presentation will be presented. Proposed approach will be presented in Section 3. Experiment setups, results and evaluations will be given in Section 4, including face recognition tests using facial skin marks alone and tests using facial skin marks combined with Eigenfaces algorithm. Finally the concluding remarks will be given in Section 5 .

\section{Study of Facial Skin Marks}

\subsection{Dataset}

No public dataset of skin marks, especially facial skin marks, was found. Instead, generic face databases have to be considered. In this work, The AR Face Database [7] is used to build a facial skin mark dataset. In this publicly available dataset, most of the subjects had two sessions of images taken, with two weeks in between these sessions, which provides possible changes of appearance in the facial skin regions of the same subject, such as changes of make-up, facial hair, facial skin irritations (for example acne, razor burns) and spectacles. Based on these changes, the characteristics of facial skin marks, especially intra-class stability, can be analyzed to a certain extent.

In this work, a facial skin mark dataset was built on the frontal images with neutral expression of each subject in the AR face database. As shown in Table 1 240 images of 120 subjects in total were included in this dataset with exactly 2 images per subject.

The automatic labeling of facial skin marks appears to be a difficult problem due to their diversity and the frequent similarity to the neighboring background pixels. In

Table 1. The facial skin marks dataset built on The AR Face Database

\begin{tabular}{|l|l|l|}
\hline & Number of subjects & Number of images \\
\hline \hline Female & 55 & 110 \\
Male & 65 & 130 \\
\hline
\end{tabular}




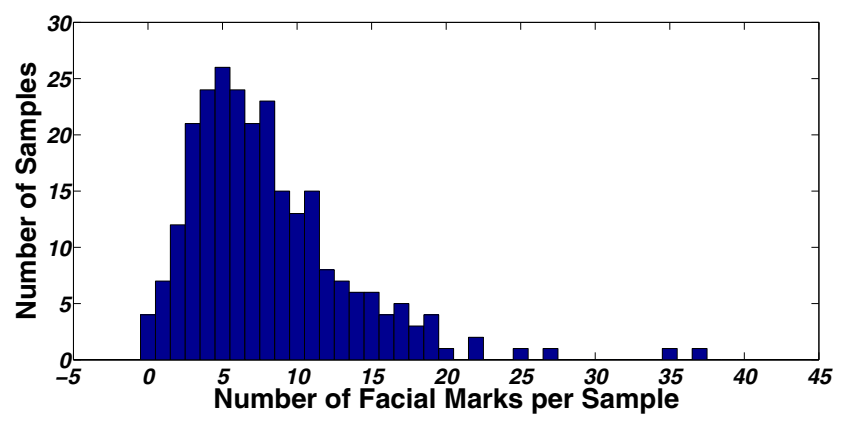

Fig. 1. Histogram of numbers of skin marks labeled for all the facial images

this work we utilize a semi-automatic approach for building facial skin mark database. First, all the images were studied by human observer closely in the full size and then one seed pixel for each facial skin mark was labeled manually. The inner eye corners of each face in the images were also marked for reference purpose. All of the 240 facial images aforementioned images were processed accordingly. Seed pixels were subsequently used for automatic extraction of features.

\subsection{Statistical Analysis}

With the ground-truthed dataset, statistical analysis was done on the characteristics of facial skin marks, especially their universality and intra-class stability.

Figure 1 shows the histogram of the number of facial skin marks in each of the available facial images. As shown in Table 2, the minimum number of facial skin marks in one facial image is 0 while the maximum number is 37 with a standard deviation of 5.5. From Figure 1, we can conclude that these extreme cases happen far less frequently compared with the cases where number of facial skin marks lies between these two numbers. The total number of facial images that contain 3 to 12 facial skin marks comprises $74.5 \%$ of all the available images. And $98.8 \%$ of all the availabe facial images have at least one facial skin mark.

Table 2. Statistics of the number of facial skin marks in all the facial images and the intra-class differences in number of facial skin marks for all the subjects

\begin{tabular}{|l|l|l|}
\hline & $\begin{array}{l}\text { Number of facial } \\
\text { skinmarks for all } \\
\text { the facial images }\end{array}$ & $\begin{array}{l}\text { Intra-class differences } \\
\text { in number of facial } \\
\text { skin marks }\end{array}$ \\
\hline \hline Min & 0 & 0 \\
Max & 37 & 10 \\
Average & 8.2 & 1.7 \\
Stdev & 5.5 & 1.7 \\
\hline
\end{tabular}


Intra-class differences are measured by calculating the differences between the number of facial skin marks of the two images from the same subject. The right column in Table 2 summaries the analysis on this matter. Within the 120 subjects, the minimum intra-class difference is 0 , while the maximum difference is 10 , with the average of 1.7 and the standard deviation of 1.7. Causes of this discrepancies could be one or more of the following conditions:

- Occlusion caused by change of

- Hair

- Facial hair

- Cosmetics

- Spectacles

- Short term changes of skin appearance caused by

- Acne

- Freckles

- Razor burns

- Environment changes such as changes of lightings.

Proper compensation can be done to eliminate environment changes to certain level. However, it is rather difficult a task to counteract the other changes since the appearance of facial skin region changes over time. Among types of facial skin marks, moles and scars are more stable than small dark regions caused by acne, freckles, razor burns or other skin irritations, however, it is difficult to recognize them completely separately since they all tend to have the similar appearance in contrast to their surrounding normal skin regions.

Although, this analysis of facial skin marks was only conducted on a dataset with limited size, it can, to some extent, be used to justify the characteristics of facial skin marks as a potential biometric trait. First, the universality of facial skin marks is satisfying with over $90 \%$ availability. In order to test the intra-class stability, experiments were conducted and results were shown in Section 4.

\subsection{Feature Presentation of Facial Skin Marks}

Based on the seed pixel labeled for each facial image, a region growing algorithm was utilized on each of region of interest that contains one of the facial skin marks, such that each of the facial skin marks would grow from one pixel into a patch containing a group of pixels that have similar intensity. After the region growing algorithm, the centers of the facial skin marks were re-calculated accordingly.

Two types of features were defined for facial skin marks, namely distance feature and local features. For distance feature, one polar coordinate system was built on each facial image, with the middle point of the two inner eye corners, $O$, as the pole and the ray from $O$ to the left corner of the right eye as polar axis. Then, the distance feature of each facial skin mark, $M_{i}$, can be represented by the polar coordinates of its newly calculated center point, $\left(r_{i}, \theta_{i}\right)$. Two local features were introduced in this work, the area of the facial skin mark, $A_{i}$, and the average intensity of all the pixels included in this skin mark, $I_{i}$, for facial skin mark $M_{i}$. 


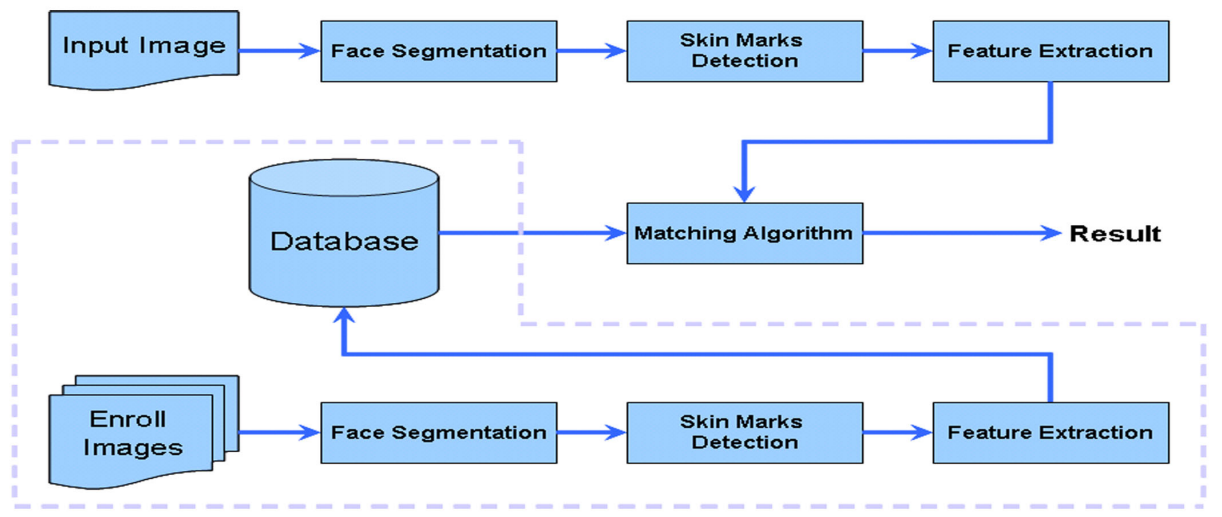

Fig. 2. The framework of personal identification system using facial skin marks as sole biometrics

\section{Facial Skin Marks as Biometric Trait}

To verify the feasibility of using facial skin marks alone to perform personal identification, one framework was proposed as shown in Figure 2. Facial images are enrolled by face segmentation, facial skin marks detection, and feature extraction for all the detected marks, then the features are stored into the database. For a probe image, the similar process would be conducted to extract its features, then the probe features would be matched against all the record in the database. Finally, the match with the highest score would be returned as identification result.

\subsection{Matching Algorithm}

Since it is possible for the images of the same subject to have different number of facial skin marks extracted, the matching of any two facial images based on facial skin marks could result in matching $N$ marks in one image against $M$ marks in the other, where $N \neq M$. To match two images, $G_{1}$ and $G_{2}$, based on their facial skin marks, one matching algorithm was proposed as following.

For the $i^{t h}$ facial skin mark in $G_{1}$, and for the $j^{\text {th }}$ facial skin mark in $G_{2}$, the weighted distance between these two facial skin marks is calculated as:

$$
D_{i, j}=\sqrt{\sum_{f=1}^{f n}\left(\omega_{f} \times \operatorname{Dist}\left(F_{i, f}, F_{j, f}\right)\right)^{2}}, \begin{aligned}
& 0 \leq i<M \\
& 0 \leq j<N
\end{aligned}
$$

In Equation $1, f n$ is the number of features for each facial skin mark, and as aforementioned, there are 3 features defined for each skin mark in this work. $\omega_{f}$ is the weight for the $f^{t h}$ feature, with $\sum_{f=1}^{f n} \omega_{f}=1$. Dist $\left(F_{i}, F_{j}\right)$ calculates the distance between two features $F_{i}$ and $F_{j}$. Depend on the type of these features, $\operatorname{Dist}\left(F_{i}, F_{j}\right)$ could calculate Euclidean distance or simple subtraction of these two features. If this $D_{i, j}$ is smaller than a preset threshold, then these two facial skin marks are considered matched. After processing all the possible matching pairs of facial skin marks in $G_{1}$ and $G_{2}$, the 


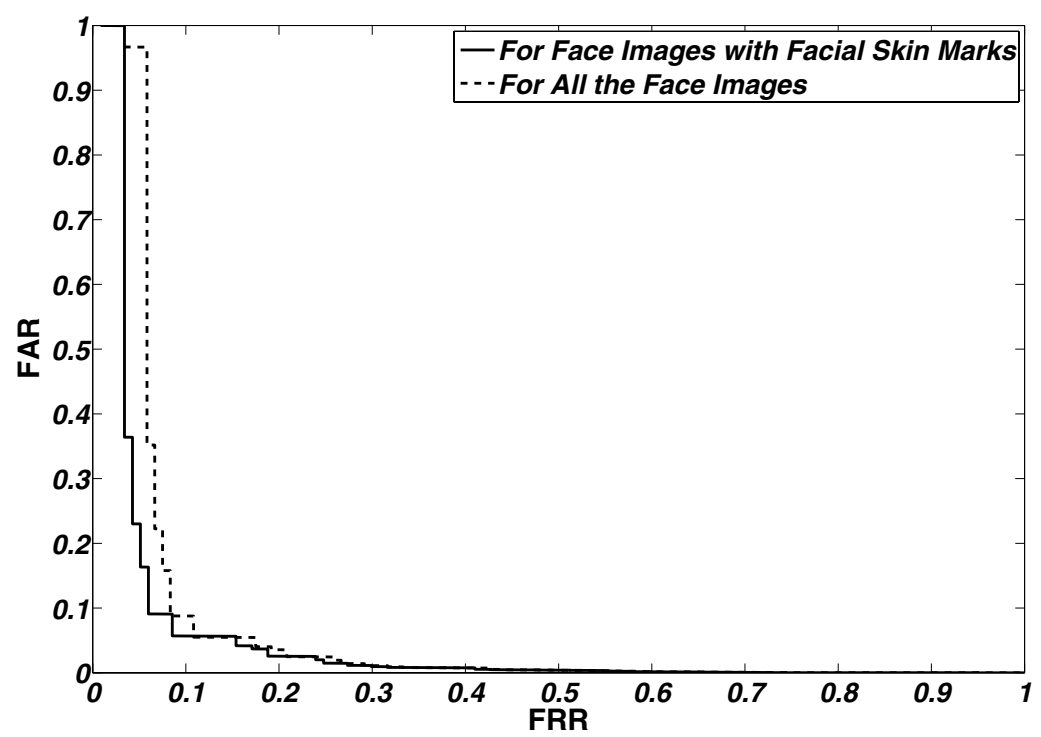

Fig. 3. ROC curves for facial skin mark matcher

number of matches, $N_{\text {matches }}$, are counted. Then the final matching score of matching these two images is calculated as:

$$
S=\frac{N_{\text {matches }}}{N_{\text {matching_pairs }}}
$$

If this score $S$ is higher than a threshold, then these two images are considered matched, otherwise not matched. Another scenario need to be considered is when at least one of the matching faces has no or too few facial skin marks detected. A flag was introduced for these cases, where the flag would be set as "FAIL". For normal cases, this flag is " $O K$ ".

\section{Experiment Results and Discussion}

Two set of experiments were conducted in this work. One set of experiment is using facial skin marks as single biometric trait to perform personal identification. The other is using the combination of facial skin marks and a conventional face recognition algorithm, in this work Eigenfaces algorithm to perform personal identification. For these two set of experiments, the same dataset, as mentioned in Section 2, was used, and of the two images of each subject, one is used as gallery image and the other as probe image.

\subsection{Facial Skin Marks as Single Biometric Trait}

In this set of experiments, facial skin marks alone was used as a biometric trait to perform personal identification. A system was build as described in Figure 2 Due to the 


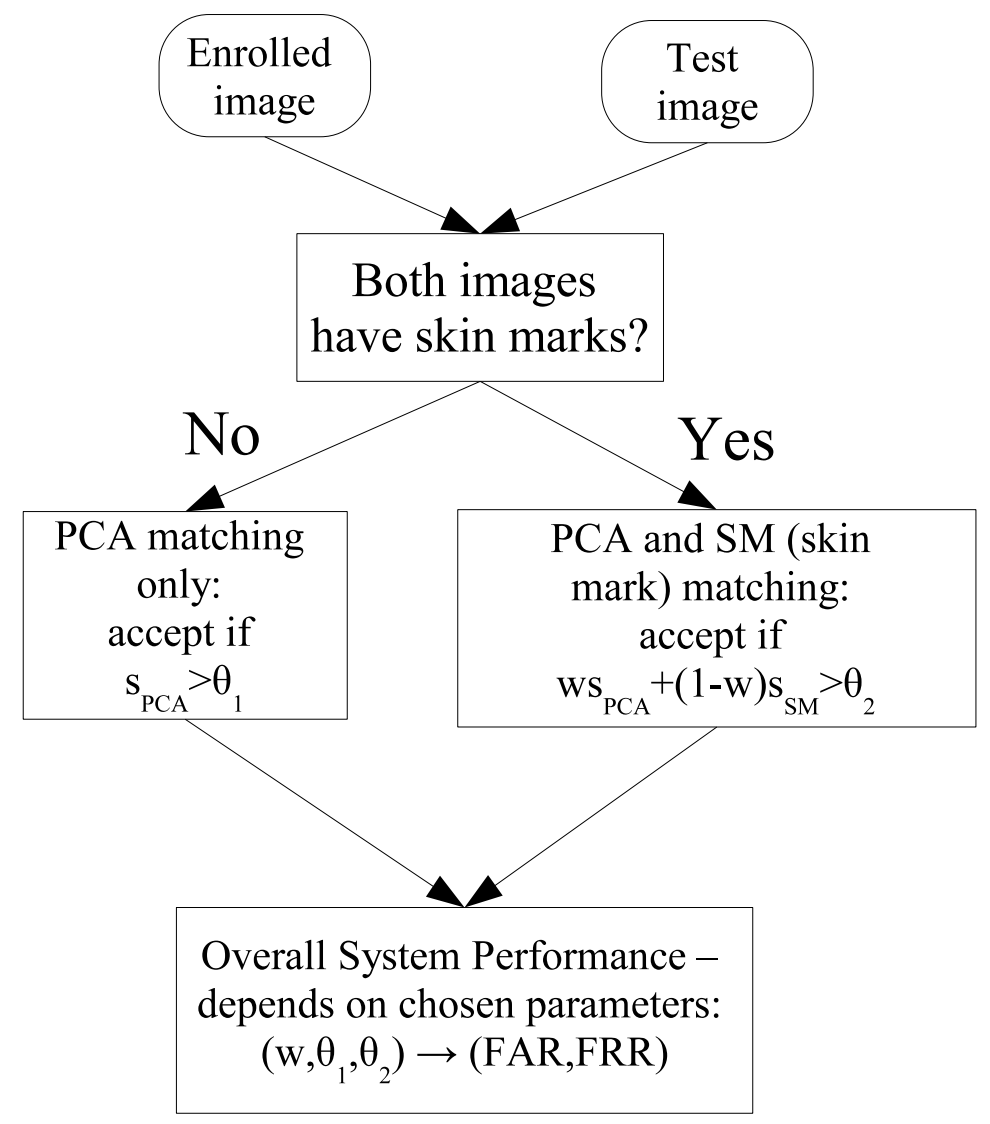

Fig. 4. Combination algorithm for branched system

nature of this trait, it is possible that the images of some of the subjects may not show any facial skin marks at all, which is the reason we introduced a flag as mention above. In order to evaluate the system under various conditions, two modes of experiments were conducted. One is to only process the images with certain number, $F_{n}$, of facial skin marks. In this work, $F_{n}$ was set to 1 . The other mode is to process all the available images, which contains both images with some facial skin marks extracted and images with no facial skin marks. With these two modes of experiments conducted on the available dataset, the results is shown in Figure 3

\subsection{Combine Facial Skin Marks with Eigenfaces}

We used the weighted sum combination method to combine the scores produced by the Eigenfaces matching and skin mark matching algorithms. Usually this combination method has reasonably good performance [8], but the weights should be trained with the help of training data. Since not all faces possess skin marks and skin mark matching 


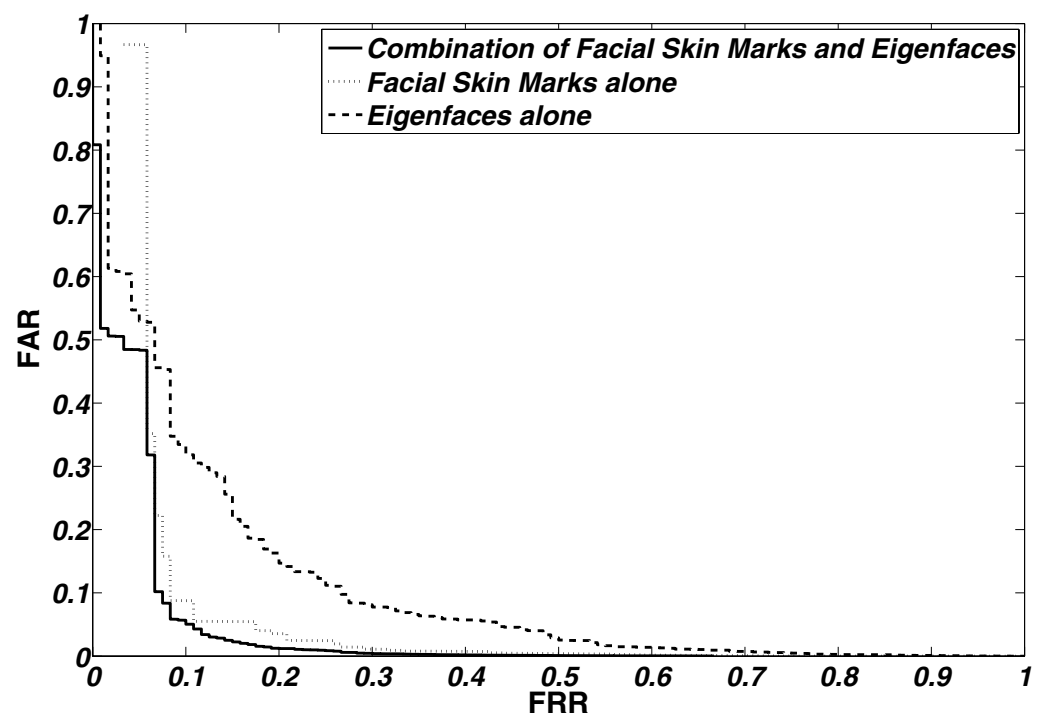

Fig. 5. ROC curves for Eigenfaces, skin mark matcher and for combined algorithm

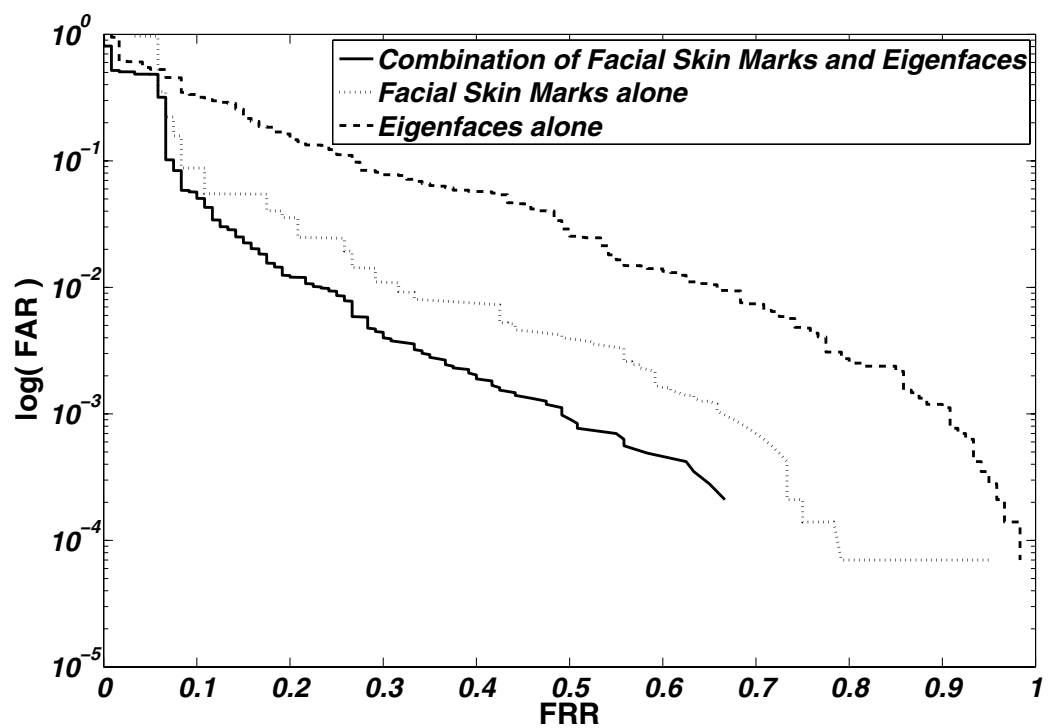

Fig. 6. ROC curves for Eigenfaces, skin mark matcher and for combined algorithm. The FAR axis was log-scaled in order to better show the performance of these algorithms for small FAR values.

score can not be calculated, we need to provide a separate processing for such cases. The general structure of the combination algorithm is shown in Figure 4

The system has two execution branches depending on whether both matched face images have skin marks detected. If there are no skin marks in any of the images we can 
only use Eigenfaces matching score and accept verification if the Eigenfaces matching score $S_{\text {Eigenfaces }}$ is better than some threshold $\theta_{1}$. If both images have skin marks, we additionally possess the skin mark matching score $S_{S M}$ and accept verification decision if the weighted sum of two scores is better than than some threshold $\theta_{2}: w S_{\text {Eigenfaces }}+$ $(1-w) S_{S M}>\theta_{2}$.

The particular choice of operating parameters $w, \theta_{1}$ and $\theta_{2}$ will result in specific values of $F A R$ and $F R R$. We call the choice of parameters $\left\{w, \theta_{1}, \theta_{2}\right\}_{0}$ locally optimal, if corresponding system's $F A R_{0}$ and $F R R_{0}$ are optimal: no other set of parameters $\left\{w, \theta_{1}, \theta_{2}\right\}_{1}$ delivers better $F A R_{1}$ and $F R R_{1}$, i.e. $F A R_{1} \leq F A R_{0}$ and $F R R_{1} \leq F R R_{0}$. The set of locally optimal values $F A R$ and $F R R$ gives us the ROC curve for optimal combinations of the type shown in Figure 4 . Note, that different points on produced ROC curve will generally have different values of parameters $w, \theta_{1}$ and $\theta_{2}$.

The presented combination algorithm is different from both serial and parallel combination architectures investigated previously in classifier combination field. It is also different from combination methods based on classifier selection methods. The difference stems from the fixed branch selection which is determined by the presence of skin marks in compared images. We can call this type of combinations as "branch combinations".

From the implementation side, the training of the presented combination method might rely on exhaustive search of locally optimal pairs $(F A R, F R R)$ with respect to system parameters $w, \theta_{1}$ and $\theta_{2}$. In our case we applied some additional optimizations for this search: efficient calculation of $F A R$ and $F R R$ based on sorted arrays of genuine and impostor matching scores and utilizing discrete nature of calculated FAR and FRR determined by the limited number of total training samples.

\subsection{Results of Combination}

Due to branching nature of combination algorithm we can analyze the performance improvements due to each branch. If compared face images have no skin marks, we rely only on Eigenfaces matcher. Consequently, we can view the combination algorithm as an improvement over skin mark matcher algorithm, especially in the area where skin mark matcher can make only reject decisions due to absence of skin marks. The areas of ROC curves with small FRR of figure 5 indicate that the performance of combined matcher is dominated by the performance of a Eigenfaces matching algorithm.

On the other hand, in the area of small FAR we can see in Figure 6 uniform improvement of combined algorithm over both Eigenfaces and skin mark matcher. This improvements can be explained by the good ability of skin mark matcher to reject false matches based on skin mark information. Such information seems to be not utilized in Eigenfaces matching algorithm.

\section{Conclusion}

This paper investigated the use of facial skin mark information for biometric person verification and presented a novel combination algorithm of conventional algorithm Eigenfaces and skin mark matcher. The resulting combined face matcher has universality property and delivers better performance than either single matcher. Future work 
comprises expanding current study of facial skin marks by incorporating more face image data, especially images with high resolution, and introducing more detailed classification and analysis of different types of facial skin marks. Study of the pattern of long term changes of facial skin marks can be useful in further evaluation of the stability of facial skin mark as a biometric trait. Facial skin marks combined with other conventional models could also be an interesting problem to investigate.

\section{References}

1. Turk, M., Pentland, A.: Face recognition using eigenfaces. In: IEEE Conf. on Computer Vision and Pattern Recognition, June 1991, pp. 586-591 (1991)

2. Belhumeur, P.N., Hespanha, J.P., Kriegman, D.J.: Eigenfaces vs. fisherfaces: recognition using class specific linearprojection. IEEE Trans. Pattern Anal. Mach. Intell. 19(7), 711-720 (1997)

3. Cootes, T.F., Edwards, G.J., Taylor, C.J.: Active appearance models. IEEE Trans. Pattern Anal. Mach. Intell. 23(6), 681-685 (2001)

4. Blanz, V., Vetter, T.: Face recognition based on fitting a 3D morphable model. IEEE Trans. Pattern Anal. Mach. Intell. 25(9), 1063-1074 (2003)

5. Lin, D., Tang, X.: Recognize high resolution faces: From macrocosm to microcosm. In: IEEE Conf. on Computer Vision and Pattern Recognition, vol. 2, pp. 1355-1362 (2007)

6. Pierrard, J.S., Vetter, T.: Skin detail analysis for face recognition. In: IEEE Conf. on Computer Vision and Pattern Recognition, June 2007, pp. 1-8 (2007)

7. Martinez, A.M., Benavente, R.: The AR Face Database. CVC Technical Report \#24 (June 1998)

8. Ivanov, Y., Serre, T., Bouvrie, J.: Error weighted classifier combination for multi-modal human identification. Technical Report AI Memo 2005-035, Massachusetts Institute of Technology Computer Science and Artificial Intelligence Laboratory (2005/12/14 (2005) 